\title{
Angular anisoplanatism in laser guide star adaptive optics
}

\author{
Marcos A. van Dam, ${ }^{a}$ Richard J. Sasiela,${ }^{b}$ Antonin H. Bouchez, ${ }^{c}$ David Le Mignant,${ }^{a}$ Randy \\ D. Campbell, ${ }^{a}$ Jason C. Y. Chin, ${ }^{a}$ Scott K. Hartman, ${ }^{a}$ Erik M. Johansson, ${ }^{a}$ Robert E. Lafon, ${ }^{a}$ \\ Paul J. Stomski, Jr., ${ }^{a}$ Douglas M. Summers ${ }^{a}$ and Peter L. Wizinowich ${ }^{a}$ \\ ${ }^{a}$ W. M. Keck Observatory, 65-1120 Mamalahoa Highway, Kamuela, HI 96743 \\ ${ }^{b}$ Current affiliation: Lincoln Laboratory, Massachussetts Institute of Technology, Lexington, \\ MA 02173 \\ ${ }^{c}$ Current affiliation: Caltech Optical Observatories, M/S 105-24, Pasadena, CA 91125
}

\begin{abstract}
The image quality obtained using laser guide star adaptive optics (LGS AO) is degraded by the fact that the wavefront aberrations experienced by light from the LGS and from the science object differ. In this paper we derive an analytic expression for the variance of the difference between the two wavefronts as a function of angular distance between the LGS and the science object. This error is a combination of focal anisoplanatism and angular anisoplanatism. We show that the wavefront error introduced by observing a science object displaced from the guide star is smaller for LGS AO systems than for natural guide star AO systems.
\end{abstract}

\section{INTRODUCTION}

Natural guide star adaptive optics (NGS AO) systems have found widespread use in astronomy to compensate for the blurring due to atmospheric turbulence. ${ }^{1}$ NGS AO suffers from two fundamental limitations. First, a sufficiently bright guide star is needed to measure the wavefront. Second, the image quality of the science target degrades with increasing distance from the guide star, an effect known as angular anisoplanatism. ${ }^{2}$ Laser guide star (LGS) AO systems circumvent the need for a bright NGS by creating an artificial star anywhere in the sky. Since the LGS cannot be used to measure wavefront tip-tilt and absolute focus, an NGS is still needed to make those measurements. However, the requirements on the magnitude of the guide star are greatly relaxed, since light from the whole aperture can be used to measure tip-tilt and the focus measurements can be made at a much lower bandwidth.

In LGS AO, there are three sources of anisoplanatism. Angular anisokinetism, also known as tip-tilt angular anisoplanatism, ${ }^{3}$ stems from the difference between the tip-tilt component of the wavefronts of the tip-tilt guide star and the science object. Focal anisoplanatism, also known as the cone effect, occurs because the LGS samples the cone of turbulence between the LGS and the telescope, while the turbulence experienced by the science object is distributed in a cylinder between itself and the telescope. Finally, angular anisoplanatism results from the difference in the wavefront terms of higher order than tip-tilt between LGS and the science object. Since the LGS can be placed anywhere on the sky, including at the same location in the sky as the science object, this error term is not fundamental. However, in practice there are usually multiple or extended objects in the field. Focal and angular anisoplanatism are intrinsically linked in LGS AO, since the wavefront sensor measures the cone of turbulence offset by an angle (which may be zero) from the cyclinder of turbulence blurring the science object. The remainder of this paper develops and evaluates the analytic expression for the isoplanatic errors due to a combination of focal and angular isoplanatism and compares it to the NGS AO isoplanatic error.

\section{ANALYTIC EXPRESSION FOR THE LGS ANISOPLANATIC ERROR}

In this section, we develop an expression for the sum of the angular and focal anisoplanatic error by propagating transverse spectral filter functions through turbulence. ${ }^{4}$ This approach has previously been used to find the errors due to LGS AO focal anisoplanatism, ${ }^{3}$ anisokinetism ${ }^{3}$ and focal anisokinetism. ${ }^{5}$ A very complicated expression for the LGS isoplanatic error, analogous to the NGS isoplanatic error, was developed by Tyler by means of

Further author information: Send correspondence to M.v.D.: E-mail: mvandam@keck.hawaii.edu 
structure function calculations. ${ }^{6}$ Here, we develop a simple relationship for LGS anisoplanatism using aperture filter functions. We want to find the aperture-averaged phase variance that results from subtracting the piston and tip-tilt removed phase of an LGS with the piston and tip-tilt removed phase of a star offset by angle $\theta$ from the LGS.

Using the analytic approach developed by Sasiela and Shelton ${ }^{7}$ and neglecting diffraction, the wavefront phase error variance, ${\sigma_{\phi}}^{2}$, can be written as

$$
\sigma_{\phi}^{2}=0.2073 k^{2} \int_{0}^{\infty} C_{n}^{2}(z)\left(\int f(\boldsymbol{\kappa}) h(\boldsymbol{\kappa}, z) \mathrm{d} \boldsymbol{\kappa}\right) \mathrm{d} z,
$$

where $k=2 \pi / \lambda$ is the wavenumber, $\lambda$ the wavelength, $C_{n}^{2}(z)$ is the refractive index structure constant at height $z$, and $\kappa=(\kappa \cos (\alpha), \kappa \sin (\alpha))$ is the spatial frequency domain coordinate. Bold face symbols are used in this paper to define two-dimensional variables, with the corresponding regular font symbol representing the amplitude and $\alpha$ the angle coordinate.

We assume a Kolmogorov turbulence power spectrum given by $f(\boldsymbol{\kappa})=\kappa^{-11 / 3}$. The final term in Eq. (1), $g(\boldsymbol{\kappa}, z)$, is the aperture filter function in the pupil plane, unique to each problem. We wish to find the squared value of the difference in a quantity between the LGS at height $L$ and an NGS at infinity offset by angle $\theta$. Neglecting diffraction, $h(\boldsymbol{\kappa}, z)$, for this problem is given by ${ }^{4}$

$$
h(\boldsymbol{\kappa}, z)=\left|G\left(\gamma_{1} \boldsymbol{\kappa}\right)-G\left(\gamma_{2} \boldsymbol{\kappa}\right) \exp \left[i \gamma_{2} \boldsymbol{\kappa} \cdot \boldsymbol{\theta} z\right]\right|^{2} .
$$

The propagation parameter $\gamma$ describes the weighting due to the convergence of the wave. For plane waves, $\gamma_{2}=1$, while for a diverging source located at height $L, \gamma_{1}=1-z / L{ }^{4}$ The filter function, $G(\gamma \boldsymbol{\kappa})$, is the Fourier transform of the aperture function which converts the phase at the aperture into a quantity of interest. If the quantity of interest is the phase, then ${ }^{4}$

$$
G_{\phi}(\gamma \boldsymbol{\kappa}, \boldsymbol{\rho})=\exp [i \gamma \boldsymbol{\kappa} \cdot \boldsymbol{\rho}]
$$

where the subscript $\phi$ denotes that the quantity of interest is the phase. There is a dependency on the aperture coordinate, $\boldsymbol{\rho}$, which we will eliminated later by averaging the phase variance over the whole aperture.

The filter functions for piston and tip-tilt are

$$
G_{\mathrm{P}}(\gamma \boldsymbol{\kappa})=\frac{2 J_{1}[\gamma \kappa D / 2]}{\gamma \kappa D / 2}
$$

and

$$
G_{\mathrm{T}}(\gamma \kappa)=\frac{4 J_{2}[\gamma \kappa D / 2]}{\gamma \kappa D / 2}
$$

respectively. ${ }^{4}$

Evaluating the difference between the phase of a star offset by angle $\theta$ from the LGS as a function of position in the aperture, we obtain

$$
\begin{aligned}
h_{\phi}(\boldsymbol{\kappa}, \boldsymbol{\rho}, z) & =|\exp [i \boldsymbol{\kappa} \cdot \boldsymbol{\rho}(1-z / L)]-\exp [i(\boldsymbol{\kappa} \cdot \boldsymbol{\rho}+\boldsymbol{\kappa} \cdot \boldsymbol{\theta} z)]|^{2} \\
& =2(1-\cos [\boldsymbol{\kappa} \cdot \boldsymbol{\rho} z / L+\boldsymbol{\kappa} \cdot \boldsymbol{\theta} z]) \\
& =2(1-\cos [\boldsymbol{\kappa} \cdot \boldsymbol{\rho} z / L] \cos [\boldsymbol{\kappa} \cdot \boldsymbol{\theta} z]+\sin [\boldsymbol{\kappa} \cdot \boldsymbol{\rho} z / L] \sin [\boldsymbol{\kappa} \cdot \boldsymbol{\theta} z]) .
\end{aligned}
$$

The last term has a sinusoidal dependence on aperture position. If the aperture is symmetric about the center, then this term averages to zero. The aperture average of the remaining terms is

$$
\begin{aligned}
h_{\phi}(\boldsymbol{\kappa}, z) & =\int \mathrm{d} \boldsymbol{\rho} h_{\phi}(\boldsymbol{\kappa}, \boldsymbol{\rho}, z) / \int \mathrm{d} \boldsymbol{\rho} \\
& =\frac{4}{\pi D^{2}} \int_{0}^{D / 2} \rho \mathrm{d} \rho \int_{0}^{2 \pi} \mathrm{d} \theta 2\left(1-\cos \left[\frac{\boldsymbol{\kappa} \cdot \boldsymbol{\rho} z}{L}\right] \cos [\boldsymbol{\kappa} \cdot \boldsymbol{\theta} z]\right)
\end{aligned}
$$




$$
\begin{aligned}
& =\frac{8}{D^{2}} \int_{0}^{D / 2} \rho \mathrm{d} \rho 2\left(1-J_{0}\left[\frac{\kappa \rho z}{L}\right] \cos [\boldsymbol{\kappa} \cdot \boldsymbol{\theta} z]\right) \\
& =2\left(1-\frac{4 L}{\kappa z D} J_{1}\left[\frac{\kappa z D}{2 L}\right] \cos [\boldsymbol{\kappa} \cdot \boldsymbol{\theta} z]\right) .
\end{aligned}
$$

The aperture averaged filter function is inserted into Eq. (1) to obtain

$$
\sigma_{\phi}^{2}=0.4146 k^{2} \int_{0}^{L} \mathrm{~d} z C_{n}^{2}(z) \int \mathrm{d} \boldsymbol{\kappa} \kappa^{-11 / 3}\left(1-\frac{4 L}{\kappa z D} J_{1}\left[\frac{\kappa z D}{2 L}\right] \cos [\boldsymbol{\kappa} \cdot \boldsymbol{\theta} z]\right) .
$$

Performing the angular integration by replacing $\int \mathrm{d} \kappa$ with $2 \pi \int \kappa \mathrm{d} \kappa$ and converting the cosine into a Bessel function of the first kind of order zero, we obtain

$$
\sigma_{\phi}^{2}=2.606 k^{2} \int_{0}^{L} \mathrm{~d} z C_{n}^{2}(z) \int_{0}^{\infty} \mathrm{d} \kappa \kappa^{-8 / 3}\left(1-\frac{4 L}{\kappa z D} J_{1}\left[\frac{\kappa z D}{2 L}\right] J_{0}[\kappa \theta z]\right) .
$$

Eq. (9) is the phase variance due to LGS anisoplanatism. However, the piston and the tip-tilt components of the LGS anisoplanatism do not affect image quality since those components are not measured using the LGS. Hence, we would like to obtain an expression for the piston and tip-tilt removed phase variance.

The filter function for the squared difference in piston between an LGS and an NGS offset by angle $\theta$ is

$$
h_{\mathrm{P}}(\boldsymbol{\kappa}, z)=\left|\frac{2 J_{1}[(1-z / L) \kappa D / 2]}{(1-z / L) \kappa D / 2}-\frac{2 J_{1}[\kappa D / 2]}{\kappa D / 2} \exp [i \boldsymbol{\kappa} \cdot \boldsymbol{\theta} z]\right|^{2} .
$$

Note that this expression does not need to be integrated over the aperture since the coefficients of the Zernike polynomials are, by definition, quantities integrated over the whole aperture. For compactness of notation, we write $a=\kappa D / 2$ and $b=(1-z / L) \kappa D / 2$. Then

$$
\begin{aligned}
\sigma_{\mathrm{P}}^{2} & =0.2073 k_{0}^{2} \int_{0}^{L} \mathrm{~d} z C_{n}^{2}(z) \int \mathrm{d} \boldsymbol{\kappa} \kappa^{-11 / 3} 4\left(\frac{J_{1}^{2}[b]}{b^{2}}+\frac{J_{1}^{2}[a]}{a^{2}}-2 \cos (\boldsymbol{\kappa} \cdot \boldsymbol{\theta} z) \frac{J_{1}[a] J_{1}[b]}{a b}\right) \\
& =5.212 k_{0}^{2} \int_{0}^{L} \mathrm{dz} C_{n}^{2}(z) \int d \kappa \kappa^{-8 / 3}\left(\frac{J_{1}^{2}[b]}{b^{2}}+\frac{J_{1}^{2}[a]}{a^{2}}-2 J_{0}(\kappa \theta z) \frac{J_{1}[a] J_{1}[b]}{a b}\right) .
\end{aligned}
$$

We can repeat this process using the tip-tilt filter function:

$$
h_{\mathrm{T}}(\boldsymbol{\kappa}, z)=\left|\frac{4 J_{2}[b]}{b}-\frac{4 J_{2}[a]}{a} \exp [i \boldsymbol{\kappa} \cdot \boldsymbol{\theta} z]\right|^{2}
$$

It follows that the tip-tilt phase variance is

$$
\begin{aligned}
\sigma_{\mathrm{T}}^{2} & =0.2073 k_{0}^{2} \int_{0}^{L} \mathrm{dz} C_{n}^{2}(z) \int d \boldsymbol{\kappa} \kappa^{-11 / 3} 16\left(\frac{J_{2}^{2}[b]}{b^{2}}+\frac{J_{2}^{2}[a]}{a^{2}}-2 \cos (\boldsymbol{\kappa} \cdot \boldsymbol{\theta} z) \frac{J_{2}[a] J_{2}[b]}{a b}\right) \\
& =20.83 k_{0}^{2} \int_{0}^{L} \mathrm{dz} C_{n}^{2}(z) \int d \kappa \kappa^{-8 / 3}\left(\frac{J_{2}^{2}[b]}{b^{2}}+\frac{J_{2}^{2}[a]}{a^{2}}-2 J_{0}[\kappa \theta z] \frac{J_{2}[a] J_{2}[b]}{a b}\right) .
\end{aligned}
$$

The effective LGS anisoplanatism is found by subtracting the piston and tip-tilt variance, Eqs. (13) and (11), from the total phase variance given in Eq. (9):

$$
\sigma_{\mathrm{EFF}}=\sigma_{\phi}^{2}-\sigma_{P}^{2}-\sigma_{T}^{2}
$$

Focal anisoplanatism is calculated simply by setting $\theta=0$ in the expression in Eq. (14). Tip-tilt focal anisoplanatism, obtained by setting $\theta=0$ in the Eq. (13), is a limitation to using a laser guide star to extract tip-tilt information. ${ }^{5}$ 
For completeness, we state the expressions for the NGS anisoplanatism used in Sect. 3. We can think of NGS anisoplanatism as a special case of LGS anisoplanatism where the distance to the guide star is set to $L=\infty$. The relationship

$$
\lim _{x \rightarrow \infty} x J_{1}\left(x^{-1}\right)=1 / 2
$$

is used to simplify the Eq. (9). The NGS total phase variance is then

$$
\sigma_{\phi, \mathrm{NGS}}^{2}=2.606 k^{2} \int_{0}^{L} \mathrm{~d} z C_{n}^{2}(z) \int_{0}^{\infty} \mathrm{d} \kappa \kappa^{-8 / 3}\left(1-J_{0}[\kappa \theta z]\right) .
$$

The effective variance due to NGS anisoplanatism is obtained by subtracting the piston component from Eq. (16):

$$
\sigma_{\mathrm{EFF}, \mathrm{NGS}}^{2}=\sigma_{\phi, \mathrm{NGS}}^{2}-\sigma_{\mathrm{P}, \mathrm{NGS}}^{2},
$$

where

$$
\sigma_{\mathrm{P}, \mathrm{NGS}}^{2}=2.606 k_{0}^{2} \int_{0}^{L} \mathrm{dz} C_{n}^{2}(z) \int d \kappa \kappa^{-8 / 3}\left(\frac{2 J_{1}[a]}{a}\right)^{2}\left(1-J_{0}[\kappa \theta z]\right) .
$$

Finally, the NGS anisokinetism, which is the tip-tilt component of the NGS anisoplanatism, is given by

$$
\sigma_{\mathrm{T}, \mathrm{NGS}}^{2}=2.606 k_{0}^{2} \int_{0}^{L} \mathrm{~d} z C_{n}^{2}(z) \int \mathrm{d} \kappa \kappa^{-8 / 3}\left(\frac{4 J_{2}[a]}{a}\right)^{2}\left(1-J_{0}[\kappa \theta z]\right) .
$$

This expression is very important since the anisoplanatic degradation in the tip-tilt correction as the angle from the tip-tilt guide star increases is the fundamental limit to the sky coverage attainable in LGS AO using a single tip-tilt guide star.

\section{NUMERICAL CALCULATION OF THE LGS ANISOPLANATIC ERROR}

In this section, we calculate numerical values for anisoplanatism for the case of the NGS and LGS AO systems on the $D=10 \mathrm{~m}$ Keck II telescope at the W. M. Keck Observatory ${ }^{8-10}$ using measured Mauna Kea turbulence profiles. The sodium layer LGS is centered at a height approximately $L=86000$ above the telescope. We use the mean of the four SCIDAR profiles presented in Tokovinin et al. ${ }^{11}$ and displayed in Table 1 in the calculations that follow. Figure 1 displays the reduction in K-band $(2.12 \mu \mathrm{m})$ Strehl ratio, $S$, due to LGS anisoplanatism

\begin{tabular}{|c|r|r|r|r|r|r|}
\hline Height $(m)$ & 500 & 1000 & 2000 & 4000 & 8000 & 16000 \\
\hline$C_{n}{ }^{2}\left(\times 10^{-14} m^{-1 / 3}\right)$ & 2.400 & 1.575 & 1.475 & 3.025 & 4.575 & 2.325 \\
\hline
\end{tabular}

Table 1. Turbulence profiles used in the calculations

as a function of distance from the LGS, obtained by evaluating Eq. (14) and using the extended Maréchal approximation, $S=\exp \left[-\sigma_{\mathrm{EFF}^{2}}{ }^{2}\right.$. The LGS anisoplanatism when the science object coincides with the LGS is due to focal anisoplanatism. The RMS wavefront error due to focal anisoplanatism was calculated to be $178 \mathrm{~nm}$, which is similar to the value, $177 \mathrm{~nm}$, calculated using Eq. (7.35) in Hardy. ${ }^{1}$

The reduction in K-band Strehl due to both the NGS anisokinetism and anisoplanatism is plotted in Fig. 2. The figure clearly illustrates that the isokinetic patch is considerably greater than the isoplanatic patch.

The component of the error due to the angular offset alone is calculated by subtracting the focal anisoplanatic contribution from the piston and tip-tilt removed anisoplanatic error. This term, which we call LGS angular anisoplanatism, is plotted in Fig. 3. We also plot the sum of the LGS angular anisoplanatism and the NGS anisokinetism, calculated using Eq. (19), since this represents the common scenario where the laser sits on top of the tip-tilt star. This is compared to the effective NGS angular anisoplanatism computed using Eq. (17).

For small angular offsets, the degradation in Strehl ratio due to anisoplanatism is much smaller if an LGS is used rather than an NGS. At a distance of 60 arcsec, however, the sum of the LGS anisoplanatism and the NGS anisokinetism is the same as the NGS anisoplanatism. 


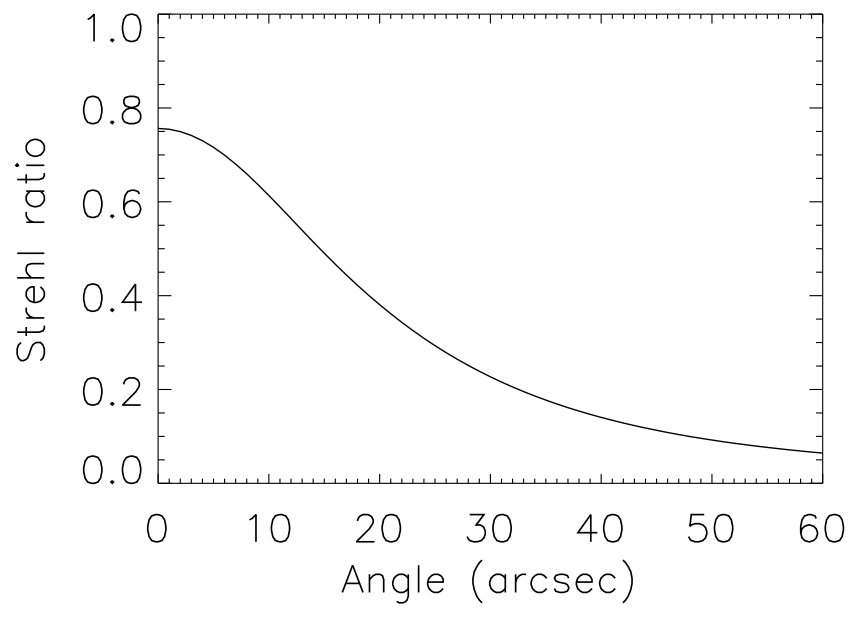

Figure 1. Reduction in K-band Strehl ratio due to LGS anisoplanatism $\left(\sigma_{\mathrm{EFF}}^{2}\right)$ as a function of angular distance from the LGS for the Mauna Kea turbulence profile.

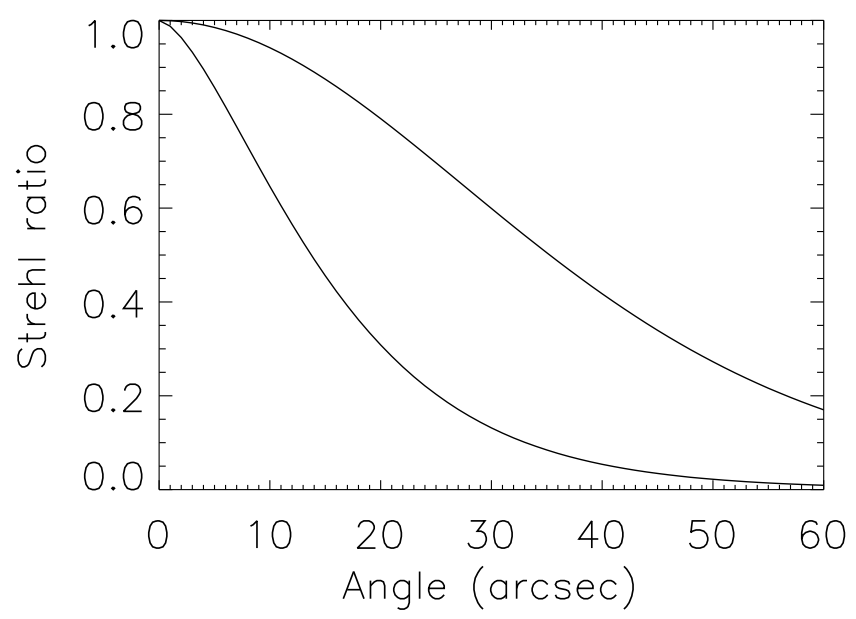

Figure 2. Reduction in K-band Strehl ratio due to, from top to bottom: NGS anisokinetism $\left(\sigma_{\text {T,NGS }}^{2}\right)$ and NGS isoplanatism $\left(\sigma_{\mathrm{EFF}, \mathrm{NGS}}^{2}\right)$ as a function of angular distance from the guide star for the Mauna Kea turbulence profile.

\section{EXPERIMENTAL RESULTS}

Several experiments were attempted on the sky to measure the anisoplanatic and anisokinetic degradation. Unfortunately, bad weather or bad and variable seeing hampered our attempts to measure this on numerous occasions. In this section, we report the techniques to measure these quantities and the measurements that we have obtained. The measurements were made using the W. M. Keck Observatory LGS AO system on the 10-m Keck II telescope. ${ }^{9,10}$

\subsection{LGS Angular Anisoplanatism}

The LGS angular anisoplanatism was measured in the following manner. A 10-mag star was acquired as a tip-tilt reference. The laser was then projected on axis, all the loops were closed and images of the tip-tilt star were 


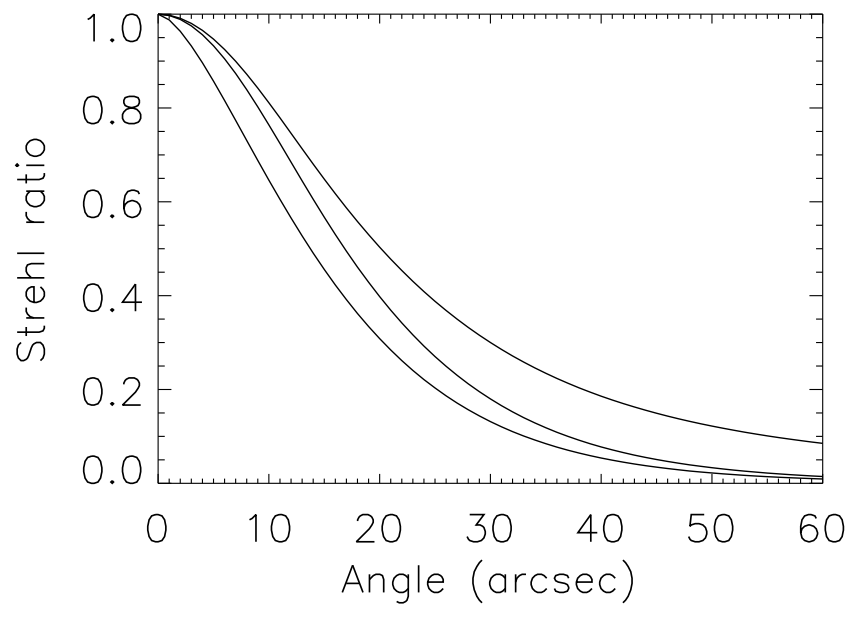

Figure 3. Reduction in K-band Strehl ratio due to, from top to bottom: LGS angular anisoplanatism $\left(\sigma_{\mathrm{EFF}}^{2}-\sigma_{\mathrm{EFF}}^{2}(0)\right)$, LGS angular anisoplanatism plus NGS anisokinetism $\left(\sigma_{\mathrm{EFF}}^{2}-\sigma_{\mathrm{EFF}}^{2}(0)+\sigma_{\mathrm{T}, \mathrm{NGS}}^{2}\right)$, NGS angular anisoplanatism $\left(\sigma_{\mathrm{EFF}, \mathrm{NGS}}^{2}\right)$

taken through a Brackett $\gamma$ filter $(2.17 \mu \mathrm{m})$ using the NIRC2 infrared camera. The position of the LGS was moved on the sky by simultaneously moving the field steering mirrors in front of the wavefront sensor and offsetting the pointing of the laser. Images of the tip-tilt guide star, which remained on axis, were captured for several different positions of the laser.

The Strehl ratio of images captured on June 30, 2005 (UT) was measured using Method 7 in Roberts et al ${ }^{12}$ and plotted in Fig. 4. We overplot the theoretical curve, $S=0.45 \exp \left[-\sigma_{\mathrm{EFF}}^{2}\right]$, using all the $C_{n}{ }^{2}$ values of the turbulence profiles in Table 1 were divided by 2.5 to fit the Strehl ratio measurements. It can be seen that there is good agreement between the measured and the theoretical curves. The measured point-spread functions are displayed in Fig. 5.

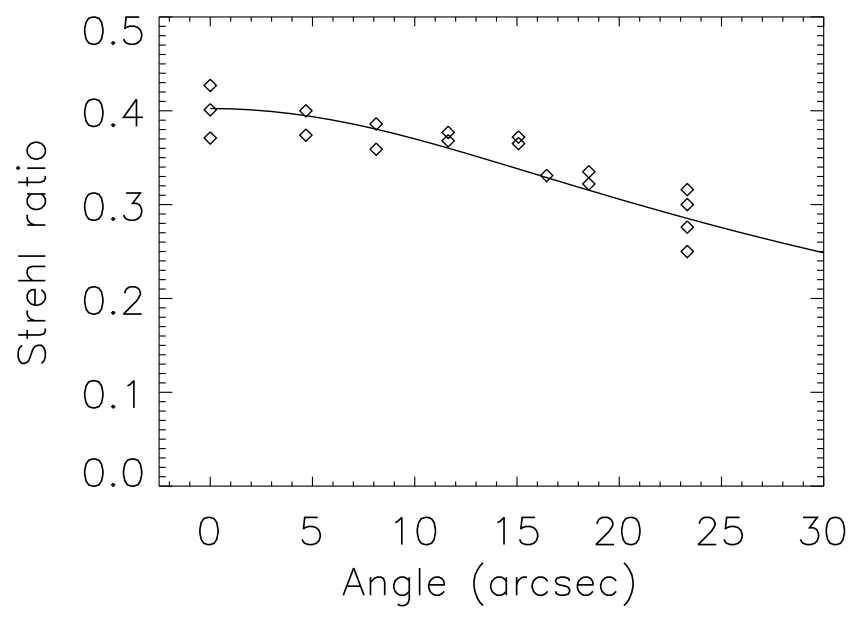

Figure 4. K-band Strehl ratio measured by moving the laser off axis. The data points represent measured values and the solid line is a best fit line using a turbulence profile with the same structure as Table 1. 

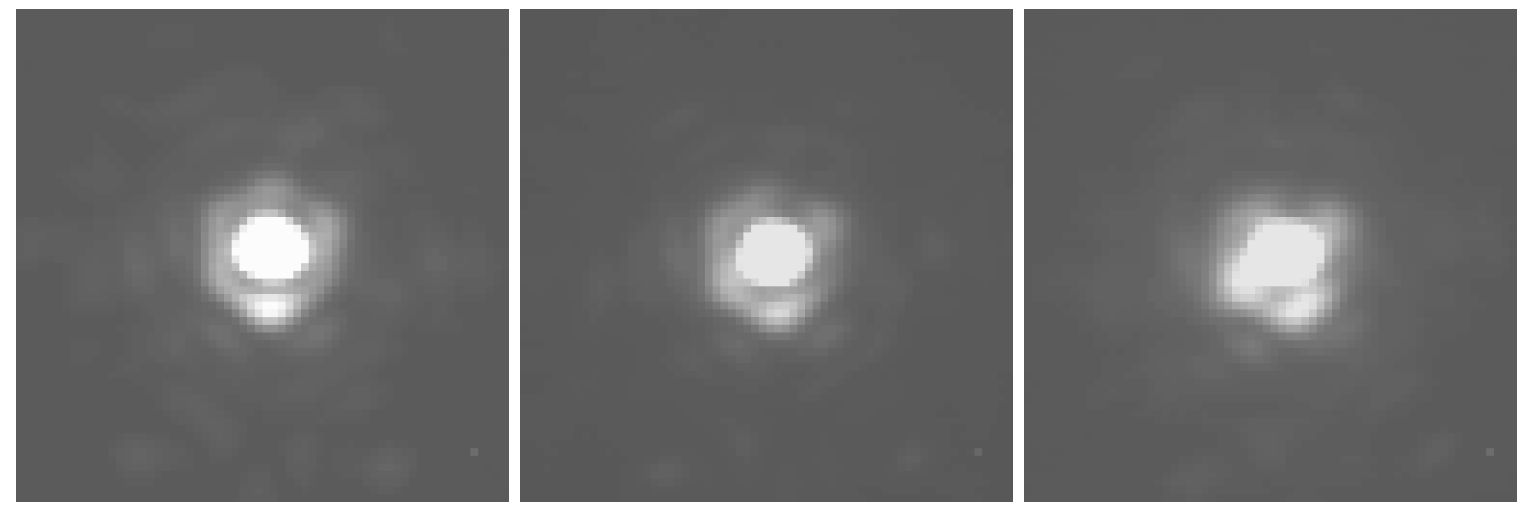

Figure 5. K-band point-spread functions measured by moving the laser (a) on axis, (b) 15 arcsec off axis, (c) 23 arcsec off axis

\subsection{NGS Angular Anisoplanatism}

We measured the NGS AO angular anisoplanatism on the Wild Duck Cluster, M11, on September 10,2005 (UT). The tip-tilt and deformable mirror loops were closed on a 11-mag star and K' $(2.12 \mu \mathrm{m})$ images were taken. Then the telescope was offset to four other stars in the cluster with the loops still closed on the same star and more images were taken. The Strehl results are displayed in Fig. 6. We also had at our disposal simultaneous turbulence profiles using the multi-aperture scintillation sensor (MASS) from the TMT site seeing characterization campaign, ${ }^{13}$ tabulated in Table 2 . The MASS instrument is not very sensitive to low-altitude turbulence, but this is not very important since anisoplanatism is dominated by high-altitude turbulence. We

\begin{tabular}{|c|r|r|r|r|r|r|}
\hline Height $(m)$ & 500 & 1000 & 2000 & 4000 & 8000 & 16000 \\
\hline$C_{n}{ }^{2}\left(\times 10^{-14} m^{-1 / 3}\right)$ & 0.000 & 0.000 & 0.000 & 0.312 & 6.940 & 0.700 \\
\hline
\end{tabular}

Table 2. Turbulence profiles measured using the MASS instrument

overplot the theoretical image degradation, $S=0.49 \exp \left[-\sigma_{\mathrm{EFF}, \mathrm{NGS}}^{2}\right]$. There is a very good fit between the measured turbulence profile and image quality. The image degradation is more benign than expected at large distances from the NGS due to the fact that the extended Maréchal approximation underestimates the Strehl ratio for large phase errors because it does not take into account the spatial correlation of the phase.

\section{CONCLUSION}

This paper presents an analytic expression for LGS AO anisoplanatism that can easily be solved using a standard mathematical software package. The focal and angular anisoplanatism in LGS AO are intrinsically linked and must be calculated together. If one subtracts the contribution due to focal anisoplanatism from this expression, one finds that the image degradation as the distance between the science object and the guide star increases is less severe for LGS AO than for NGS AO. In future work, we hope to obtain more anisoplanatism and anisokinetism measurements and compare them with theoretical predictions using measured turbulence profiles from the MASS instrument.

\section{ACKNOWLEDGMENTS}

We wish to thank Matthias Schöck and Tony Travouillon for providing the TMT MASS data. The data presented herein were obtained at the W. M. Keck Observatory, which is operated as a scientific partnership among the California Institute of Technology, the University of California, and the National Aeronautics and Space Administration. The Observatory was made possible by the generous financial support of the W. M. Keck Foundation. The authors wish to recognize and acknowledge the significant cultural role and reverence that the summit of Mauna Kea has always had within the Hawaiian community. We are most fortunate to have the 


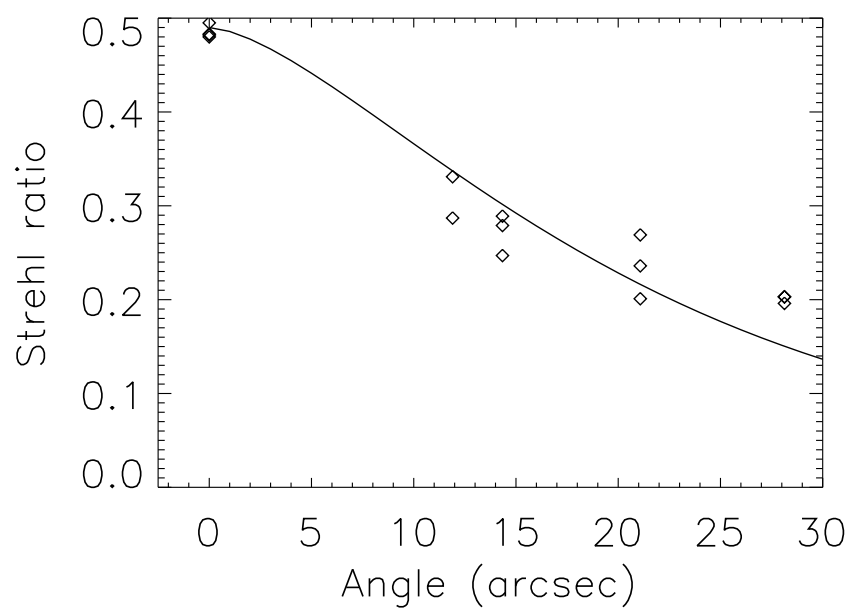

Figure 6. Measured and modeled K-band Strehl ratio as a function of distance from the NGS

opportunity to conduct observations from this mountain. This work has been supported by the National Science Foundation Science and Technology Center for Adaptive Optics, managed by the University of California at Santa Cruz under cooperative agreement No. AST - 9876783.

\section{REFERENCES}

1. J. W. Hardy, Adaptive Optics for Astronomical Telescopes (Oxford University Press, New York, 1998).

2. D. L. Fried, "Anisoplanatism in adaptive optics," J. Opt. Soc. Am. 72, 52-61 (1982).

3. R. R. Parenti and R. J. Sasiela, "Laser-guide-star systems for astronomical applications," J. Opt. Soc. Am. A 11, 288 (1994).

4. R. J. Sasiela, Electromagnetic wave propagation in turbulence: evaluation and application of Mellin transforms (Springer-Verlag, Berlin, 1994).

5. C. R. Neyman, "Focus anisoplanatism: a limit to the determination of tip-tilt with laser guide stars," Optics Letters 21, 1806-1808 (1996).

6. G. A. Tyler, "Wave-front compensation for imaging with off-axis guide stars," J. Opt. Soc. Am. A 11, 339-346 (1994).

7. R. J. Sasiela and J. D. Shelton, "Transverse spectral filtering and Mellin transform techniques applied to the effect of outer scale on tilt and tilt anisoplanatism," J. Opt. Soc. Am. A 10, 646-660 (1993).

8. M. A. van Dam, D. Le Mignant and B. A. Macintosh, "Performance of the Keck Observatory adaptive-optics system," Applied Optics 43, 5458-5467 (2004).

9. P. L. Wizinowich, D. Le Mignant, A. H. Bouchez, R. D. Campbell, J. C. Y. Chin, A. R. Contos, M. A. van Dam, S. K. Hartman, E. M. Johansson, R. E. Lafon, R. E., H. Lewis, P. J. Stomski, D. M. Summers, C. G. Brown, P. M. Danforth, and D. M. Pennington, "The W. M. Keck Laser Guide Star Adaptive Optics System: Overview," Pub. Astron. Soc. Pac. 118, 297-309 (2006).

10. M. A. van Dam, A. H. Bouchez, D. Le Mignant, E. M. Johansson, P. L. Wizinowich, R. D. Campbell, J. C. Y. Chin, S. K. Hartman, R. E. Lafon, P. J. Stomski, Jr. and D. M. Summers, "The W. M. Keck Observatory Laser Guide Star Adaptive Optics System: Performance Characterization," Pub. Astron. Soc. Pac. 118, 310-318 (2006).

11. A. Tokovinin, J. Vernin, A. Ziad and M. Chun, "Optical turbulence profiles at Mauna Kea measured by MASS and SCIDAR," Pub. Astron. Soc. Pac. 117, 395-400 (2004).

12. L. C. Roberts, Jr, J. C. Christou, R. B. Makidon, A. Sivaramakrishnan, M. D. Perrin, F. Marchis, M. Troy, B. A. Macintosh, L. A. Poyneer, M. A. van Dam, "How to compute Strehl ratio," submitted to PASP (2006). 
13. W. Skidmore, M. Schöck, A. A. Tokovinin, G. Djorgoski, A. R. Walker, R. D. Blum, T. Travouillon, J. Seguel, E. E. Bustos, D. Walker, J. Vasquez and P. E. Gillett, "The Thirty Meter Telescope site testing system,"Proc. SPIE 5489, 154-164 (2004). 